\title{
Staggered domain wall fermions
}

\section{Christian Hoelbling*}

Department of Physics, University of Wuppertal, D-42119 Wuppertal, Germany

E-mail: hcheuni-wuppertal.de

\section{Christian Zielinski}

Division of Mathematical Sciences, Nanyang Technological University, Singapore 637371 \&

Department of Physics, University of Wuppertal, D-42119 Wuppertal, Germany

E-mail: zielinski@pmail.ntu.edu.sg

We construct domain wall fermions with a staggered kernel and investigate their spectral and chiral properties numerically in the Schwinger model. In some relevant cases we see an improvement of chirality by more than an order of magnitude as compared to usual domain wall fermions. Moreover, we present first results for four-dimensional quantum chromodynamics, where we also observe significant reductions of chiral symmetry violations for staggered domain wall fermions.

34th annual International Symposium on Lattice Field Theory

24-30 July 2016

University of Southampton, UK

${ }^{*}$ Speaker. 


\section{Introduction}

When implementing chiral fermions on the lattice, domain wall fermions $[1,2,3]$ are a wellknown alternative to the computationally very expensive overlap construction $[4,5,6]$. By means of massive interacting fermions in $d+1$ dimensions, one can formulate lattice fermions with an approximate chiral symmetry in $d$ dimensions. The accuracy of this approximate symmetry is controlled by the extent of the extra dimension, where the limit of an infinite extent can be concisely described by an overlap operator. Domain wall fermions are computationally cheaper, permitting a simpler approach to parallelization and allowing for an easier tunneling between different topological sectors as compared to overlap fermions. This, however, comes at the price of replacing the exact chiral symmetry of overlap fermions with an approximate one.

Traditionally, domain wall fermions are used with a Wilson kernel as the naïve application of the formalism to the staggered kernel fails due to the lack of some technical properties within the staggered framework. This problem was eventually overcome with Adams' proposal of staggered domain wall fermions $[7,8]$, where one introduces a modified kernel operator, i.e. so-called staggered Wilson fermions. The resulting novel kernel operator is constructed by adding a suitable flavored mass term [9] to staggered fermions (see also Ref. [10]). As staggered Wilson fermions appear to be computationally more efficient than the Wilson kernel [11], one can hope for a computationally cheaper and possibly more chiral formulation. Besides staggered domain wall fermions, staggered Wilson fermions also permit the formulation of staggered overlap fermions [12, 13] with a welldefined index [14].

In this report, we present selected results of our investigations of the spectral properties and chiral symmetry violations of domain wall fermions with Wilson and staggered Wilson kernel operators in the Schwinger model [15] as recently discussed in Ref. [16]. In addition, we present first results for the case of four-dimensional quenched quantum chromodynamics.

\section{Formulation}

We begin with a quick review of the $d$-dimensional kernel operators and the $(d+1)$-dimensional domain wall fermion construction $(d=2,4)$. We denote the lattice spacing by $a$ in the first $d$ dimensions and by $a_{d+1}$ in the extra dimension. The $\gamma_{\mu}$ matrices $(\mu=1, \ldots, d)$ refer to a representation of the Dirac algebra, where we use the notation $\gamma_{d+1}$ for the chirality matrix.

Kernel operators. We denote the $d$-dimensional Wilson Dirac operator with bare fermion mass $m_{\mathrm{f}}$ by $D_{\mathrm{w}}\left(m_{\mathrm{f}}\right)$. For the definition of staggered Wilson fermions, we use our notation introduced in Ref. [16] and write the Dirac operator as $D_{\mathrm{sw}}\left(m_{\mathrm{f}}\right)=D_{\mathrm{st}}+m_{\mathrm{f}}+W_{\mathrm{st}}$. Here $D_{\mathrm{st}}=\eta_{\mu} \nabla_{\mu}$ is the usual staggered Dirac operator and our choice of the staggered Wilson term can be compactly written as

$$
W_{\mathrm{st}}=\frac{r}{a}\left(\mathbb{1}+\lambda W_{\mathrm{st}}^{1 \cdots d}\right), \quad \lambda=(-1)^{\frac{d+2}{2}} .
$$

In four dimensions our $W_{\text {st }}$ equals Adams' staggered Wilson term, for the two-dimensional case we refer to our discussion in Ref. [16]. In the following discussion, we set the $d$-dimensional lattice spacing to $a=1$ and the (staggered) Wilson parameter to $r=1$. 


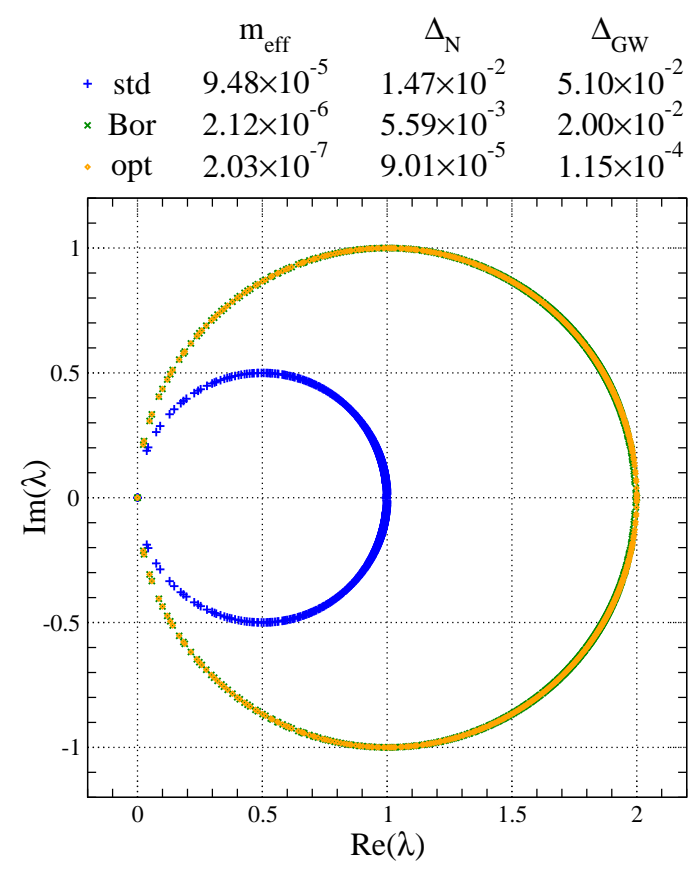

(a) Wilson kernel

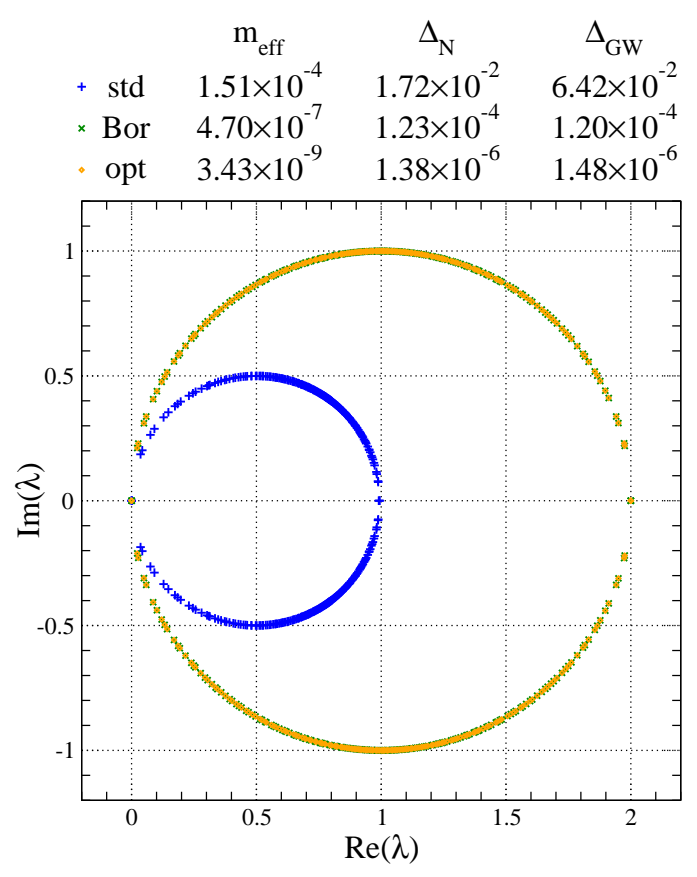

(b) Staggered Wilson kernel

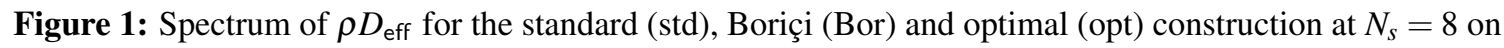
a $20^{4}$ configuration with topological charge $Q=3$ in the Schwinger model at $\beta=5$.

Bulk operators. The bulk Dirac operator in the standard (i.e. original) formulation of domain wall fermions reads

$$
\bar{\Psi} D_{\mathrm{dw}} \Psi=\sum_{s=1}^{N_{s}} \bar{\Psi}_{s}\left[D_{\mathrm{w}}^{+} \Psi_{s}-P_{-} \Psi_{s+1}-P_{+} \Psi_{s-1}\right]
$$

where the extent of the extra dimension is denoted by $N_{s}$, the $(d+1)$-dimensional fermion fields $\bar{\Psi}$ and $\Psi$ have an index $s$ for the additional spatial coordinate, we introduced $D_{\mathrm{w}}^{ \pm}=a_{d+1} D_{\mathrm{w}}\left(-M_{0}\right) \pm \mathbb{1}$, the domain wall height is given by $M_{0}$ and the chiral projectors are defined as $P_{ \pm}=\left(\mathbb{1} \pm \gamma_{d+1}\right) / 2$. In the extra dimension we impose the boundary conditions

$$
P_{+}\left(\Psi_{0}+m \Psi_{N_{s}}\right)=0, \quad P_{-}\left(\Psi_{N_{s}+1}+m \Psi_{1}\right)=0,
$$

where the fermion mass is controlled by the parameter $m$. Finally, by letting $q=P_{+} \Psi_{N_{s}}+P_{-} \Psi_{1}$ and $\bar{q}=\bar{\Psi}_{1} P_{+}+\bar{\Psi}_{N_{s}} P_{-}$, we can define $d$-dimensional fermion fields from the boundary.

Besides this original formulation, we also consider two common $\mathcal{O}\left(a_{d+1}\right)$ modifications. Boriçi's construction [17] follows from the replacement

$$
P_{+} \Psi_{s-1} \rightarrow-D_{\mathrm{w}}^{-} P_{+} \Psi_{s-1}, \quad P_{-} \Psi_{s+1} \rightarrow-D_{\mathrm{w}}^{-} P_{-} \Psi_{s+1},
$$

while Chiu introduces additional weight factors for the formulation of optimal domain wall fermions [18] in order to improve chiral properties.

Effective operators. Following the derivation given in Refs. [19, 17] and integrating out $N_{s}-1$ heavy modes, we can define a $d$-dimensional low-energy effective action $S_{\text {eff }}=\sum_{x} \bar{q}(x) D_{\text {eff }} q(x)$, 
where $D_{\text {eff }}^{-1}(x, y)=\langle q(x) \bar{q}(y)\rangle$. Note that in order to cancel the diverging contributions from the heavy fermions in the chiral limit $N_{s} \rightarrow \infty$, one introduces suitable pseudofermion fields. The effective operator can then be written in closed form as

$$
D_{\text {eff }}=\frac{1+m}{2} \mathbb{1}+\frac{1-m}{2} \gamma_{d+1} \frac{T_{+}^{N_{s}}-T_{-}^{N_{s}}}{T_{+}^{N_{s}}+T_{-}^{N_{s}}},
$$

where $T_{ \pm}=\mathbb{1} \pm a_{d+1} H$. In the case of the standard construction $H$ equals the modified kernel operator $H_{\mathrm{m}}=\gamma_{d+1} D_{\mathrm{m}}\left(-M_{0}\right)$ with $D_{\mathrm{m}}=D_{\mathrm{w}} /\left(2 \cdot \mathbb{1}+a_{d+1} D_{\mathrm{w}}\right)$. For Boriçi's construction, we find the standard kernel $H_{\mathrm{w}}=\gamma_{d+1} D_{\mathrm{w}}\left(-M_{0}\right)$ and that the effective Dirac operator equals the polar decomposition approximation of Neuberger's overlap operator. Finally, for Chiu's optimal construction the fraction on the right hand side of Eq. (2.5) is replaced by Zolotarev's optimal rational function approximation [20] of $\operatorname{sign} H_{\mathrm{w}}$. In all cases the corresponding overlap operator is given by $D_{\text {ov }}=\lim _{N_{s} \rightarrow \infty} D_{\text {eff }}$.

As discussed in full detail in Ref. [16], in order to ensure the same scale of all effective operators, we let $\rho=2 \omega$ with

$$
\omega= \begin{cases}M_{0}-\frac{1}{2} a_{d+1} M_{0}^{2} & \text { for the standard construction, } \\ M_{0} & \text { for Boriçi's/Chiu's construction }\end{cases}
$$

and consider $\rho D_{\text {eff }}$ and $\rho D_{\text {ov }}$ in all our numerical investigations.

Staggered versions. As shown in Ref. [8], one can obtain a staggered version of domain wall fermions by means of a replacement rule. In our setting, it takes the form $D_{\mathrm{w}} \rightarrow D_{\mathrm{sw}}, \gamma_{d+1} \rightarrow \varepsilon$ with $\varepsilon(x)=(-1)^{x_{1} / a+\cdots+x_{d} / a}$, allowing a full generalization of the previous discussion to the staggered case.

\section{Numerical results}

For the numerical part of our work, we begin by introducing measures of chirality followed by the discussion of selected results in the setting of the Schwinger model and four-dimensional quantum chromodynamics. For the remainder of this report, we consider the massless case $m=0$.

Measures of chirality. In order to quantify chiral symmetry violations for the effective Dirac operators, we define three different measures. The first is the effective mass $m_{\text {eff }}$ as used in Ref. [21]. Using periodic boundary conditions and considering a topologically nontrivial configuration, we let

$$
m_{\text {eff }}=\min _{\lambda \in \operatorname{spec} H}|\lambda|=\min _{\Lambda \in \operatorname{spec} D^{\dagger} D} \sqrt{\Lambda} .
$$

As chiral properties imply the normality of the Dirac operator [22], we also consider deviations from operator normality as given by $\Delta_{\mathrm{N}}=\left\|\left[D, D^{\dagger}\right]\right\|_{\infty}$. Finally, we can directly measure violations of the Ginsparg-Wilson relation by

$$
\Delta_{\mathrm{GW}}=\left\|\left\{\gamma_{d+1}, D\right\}-\omega^{-1} D \gamma_{d+1} D\right\|_{\infty},
$$

where in the staggered case $\gamma_{d+1}$ is replaced by $\varepsilon$. We note that for the overlap operator, all our measures vanish in exact arithmetics. 


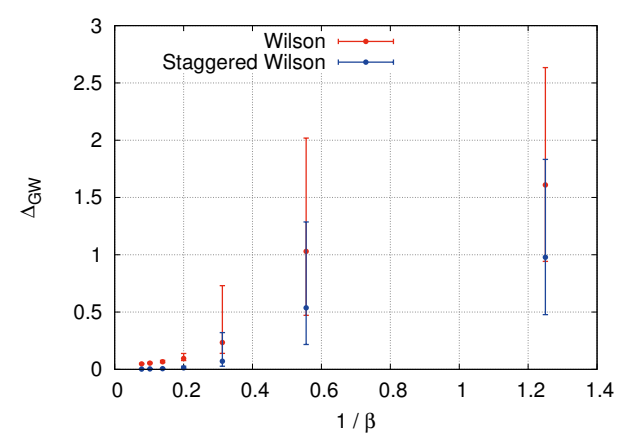

(a) Without smearing

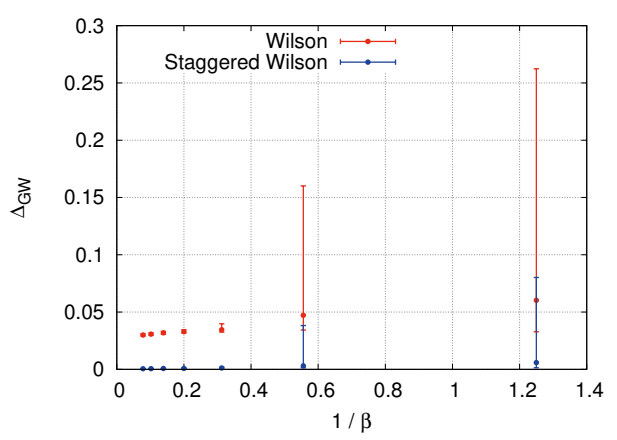

(b) With smearing

Figure 2: Measure $\Delta_{\mathrm{GW}}$ for $\rho D_{\text {eff }}$ in the optimal construction at $N_{s}=4$ in the Schwinger model.

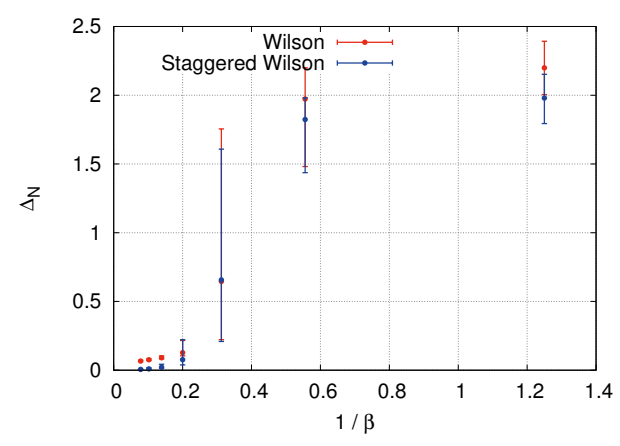

(a) Without smearing

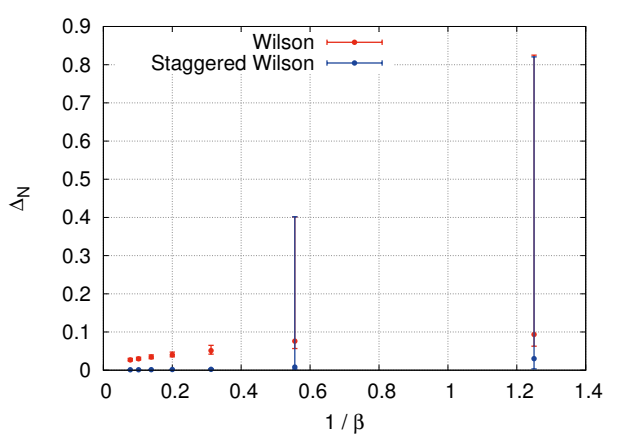

(b) With smearing

Figure 3: Measure $\Delta_{\mathrm{N}}$ for $\rho D_{\text {eff }}$ in Boriçi's construction at $N_{s}=4$ in the Schwinger model.

Schwinger model. Let us now discuss a few selected results for the two-dimensional Schwinger model. For our numerical study we use the canonical choice $M_{0}=1$.

In Fig. 1, we find the eigenvalue spectra of the effective operator $\rho D_{\text {eff }}$ for various constructions on an exemplary gauge configuration. We observe that already for the small value of $N_{s}=8$ the spectrum very closely resembles that of the corresponding overlap operator. Comparing the effective operators with the usual Wilson and the staggered Wilson operator, we note that in the case of the standard construction $m_{\mathrm{eff}}, \Delta_{\mathrm{N}}$ and $\Delta_{\mathrm{GW}}$ are typically of comparable magnitude. For Boriçi's and Chiu's optimal construction, however, we find that the staggered construction shows notably improved chiral properties compared to the Wilson case.

We are particularly interested in the chiral properties when approaching the continuum limit. To this end we generated seven ensembles with the setup of Ref. [23] with $10^{3}$ configurations each, namely $8^{2}$ at $\beta=0.8,12^{2}$ at $\beta=1.8,16^{2}$ at $\beta=3.2,20^{2}$ at $\beta=5.0,24^{2}$ at $\beta=7.2,28^{2}$ at $\beta=9.8$ and $32^{2}$ at $\beta=12.8$. The values for $\beta$ were chosen so that the physical volume is kept fixed. We consider both unsmeared and three-step APE smeared configurations [24] with a smearing parameter of $\alpha=0.5$, resulting in 14000 configurations in total.

In Figs. 2 and 3, we find two examples for the behavior of $\Delta_{\mathrm{GW}}$ and $\Delta_{\mathrm{N}}$ when $\beta$ is varied. In the figures, we plot the median value and the $68.3 \%$-width of the distribution. We find that domain wall fermions with a staggered Wilson kernel show clearly superior chiral properties in the Schwinger 


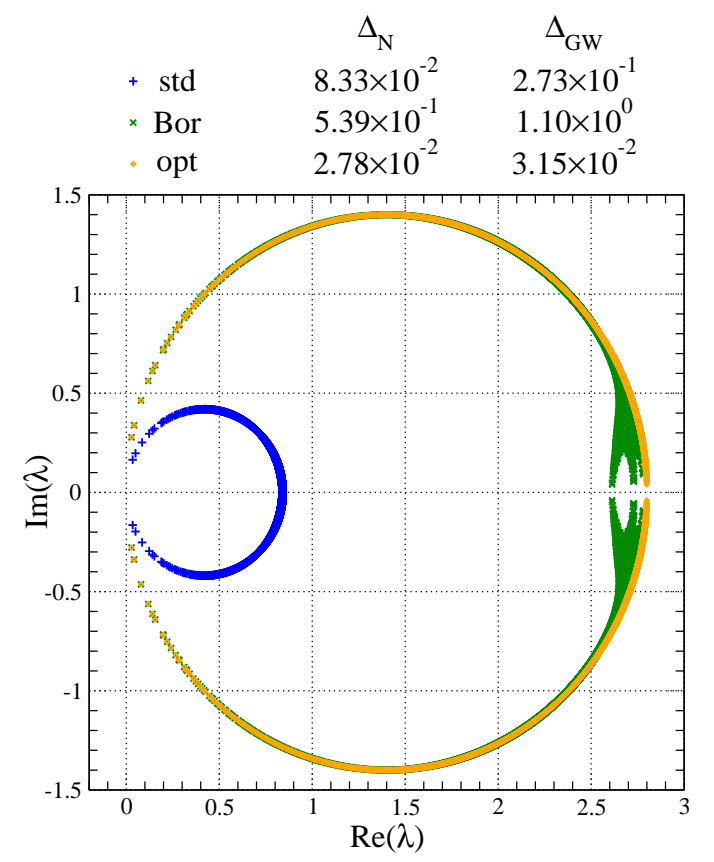

(a) Wilson kernel

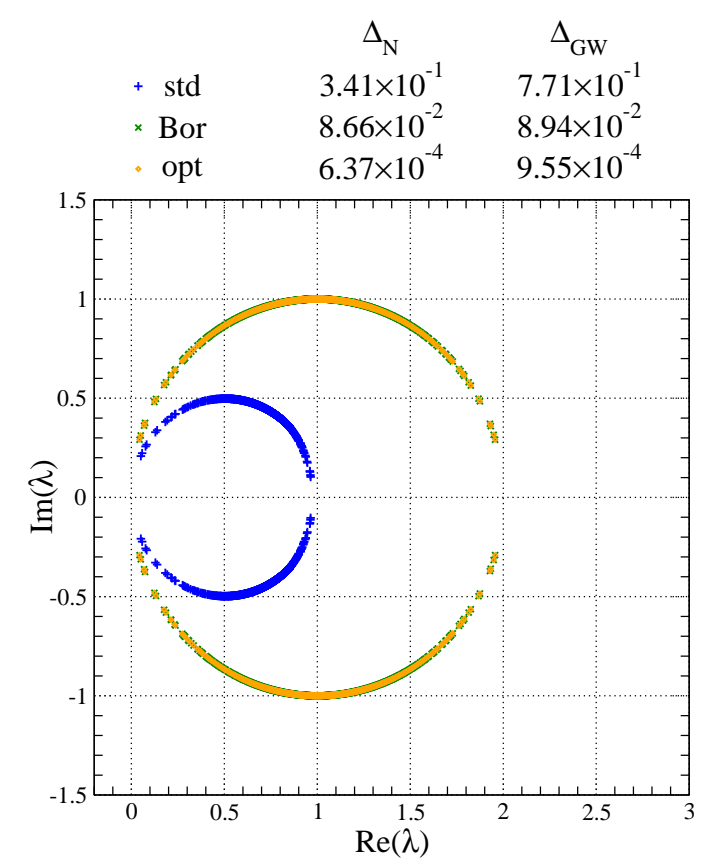

(b) Staggered Wilson kernel

Figure 4: Spectrum of $\rho D_{\text {eff }}$ with a Wilson kernel and a staggered Wilson kernel at $N_{s}=8$ in $\mathrm{QCD}_{4}$ at $\beta=6$.

model in the limit of large $\beta$, where in some cases chiral symmetry violations are reduced by more than an order of magnitude.

Quenched quantum chromodynamics. Going beyond our study in Ref. [16], we finally discuss first results for staggered domain wall fermions in the setting of quenched quantum chromodynamics in four dimensions. In Fig. 4, we show the eigenvalue spectrum of the standard, Boriçi's and the optimal construction at $N_{s}=8$ on a smeared $6^{4}$ configuration at $\beta=6$. The configuration was smeared with one APE smearing iteration using $\alpha=0.65$. For Wilson fermions we use a domain wall height of $M_{0}=1.4$, while for staggered Wilson fermions $M_{0}=1$ remains the canonical choice. As we are dealing with a topologically trivial configuration, we omit $m_{\mathrm{eff}}$ in the figure labels.

Although it is too early for a full assessment of the chiral properties of the effective operators without obtaining more statistics, we note that on the present gauge configuration we observe a large reduction of $\Delta_{N}$ and $\Delta_{\mathrm{GW}}$ by typically more than an order of magnitude for Boriçi's and the optimal construction when using the staggered Wilson kernel. For the standard construction, however, the Wilson-based construction shows better chiral properties.

\section{Conclusions}

The use of staggered domain wall fermions results in significantly improved chiral properties in the Schwinger model, offering the prospect of a computationally cheaper construction. In the setting of four-dimensional quantum chromodynamics, our first results are also very encouraging and warrant further investigations. 
Acknowledgments. C. H. is supported by DFG grant SFB/TRR-55. C. Z. is supported by the Singapore International Graduate Award (SINGA) and Nanyang Technological University.

\section{References}

[1] D. B. Kaplan, Phys. Lett. B288, 342 (1992), arXiv:hep-lat/9206013 [hep-lat] .

[2] Y. Shamir, Nucl. Phys. B406, 90 (1993), arXiv:hep-lat/9303005 [hep-lat] .

[3] V. Furman and Y. Shamir, Nucl. Phys. B439, 54 (1995), arXiv:hep-lat/9405004 [hep-lat] .

[4] P. H. Ginsparg and K. G. Wilson, Phys. Rev. D25, 2649 (1982).

[5] P. Hasenfratz, V. Laliena, and F. Niedermayer, Phys. Lett. B427, 125 (1998), arXiv:heplat/9801021 [hep-lat] .

[6] M. Luscher, Phys. Lett. B428, 342 (1998), arXiv:hep-lat/9802011 [hep-lat] .

[7] D. H. Adams, Phys. Rev. Lett. 104, 141602 (2010), arXiv:0912.2850 [hep-lat] .

[8] D. H. Adams, Phys. Lett. B699, 394 (2011), arXiv:1008.2833 [hep-lat] .

[9] M. F. L. Golterman and J. Smit, Nucl. Phys. B245, 61 (1984).

[10] P. de Forcrand, A. Kurkela, and M. Panero, JHEP 04, 142 (2012), arXiv:1202.1867 [hep-lat] .

[11] D. H. Adams, D. Nogradi, A. Petrashyk, and C. Zielinski, PoS LATTICE2013, 353 (2014), arXiv:1312.3265 [hep-lat] .

[12] D. H. Adams, PoS LATTICE2010, 073 (2010), arXiv:1103.6191 [hep-lat] .

[13] P. de Forcrand, A. Kurkela, and M. Panero, PoS LATTICE2010, 080 (2010), arXiv:1102.1000 [hep-lat] .

[14] D. H. Adams, R. Har, Y. Jia, and C. Zielinski, PoS LATTICE2013, 462 (2014), arXiv:1312.7230 [hep-lat] .

[15] J. S. Schwinger, Phys. Rev. 128, 2425 (1962).

[16] C. Hoelbling and C. Zielinski, Phys. Rev. D94, 014501 (2016), arXiv:1602.08432 [hep-lat] .

[17] A. Borici, Nucl. Phys. Proc. Suppl. 83, 771 (2000), arXiv:hep-lat/9909057 [hep-lat] .

[18] T.-W. Chiu, Phys. Rev. Lett. 90, 071601 (2003), arXiv:hep-lat/0209153 [hep-lat] .

[19] Y. Kikukawa and T. Noguchi, Nuclear Physics B - Proceedings Supplements 83, 630 (2000), arXiv:hep-lat/9902022 [hep-lat] .

[20] E. I. Zolotarev, Zap. Imp. Akad. Nauk. St. Petersburg 30, 1 (1877), reprinted in his Collected Works, Vol. II, Izdat. Akad. Nauk SSSR, Moscow, 1932, pp. 1-59. In Russian.

[21] V. Gadiyak, X.-D. Ji, and C.-W. Jung, Phys. Rev. D62, 074508 (2000), arXiv:hep-lat/0002023 [hep-lat] .

[22] W. Kerler, Chinese Journal of Physics 38, 623 (2000).

[23] S. Durr and C. Hoelbling, Phys. Rev. D69, 034503 (2004), arXiv:hep-lat/0311002 [hep-lat] .

[24] M. Falcioni, M. L. Paciello, G. Parisi, and B. Taglienti, Nucl. Phys. B251, 624 (1985). 